\title{
Gestión tecnológica en el proceso de relación Universidad del Zulia-Sector Productivo
}

\author{
Martínez de Carrasquero, Cynthia* \\ González de Gutiérrez, Marié** \\ Rojas de Mavárez, Ligibther***
}

\section{Resumen}

El propósito de este estudio es el proporcionar propuestas de gestión tecnológica, que aplicadas a las dependencias universitarias de LUZ, permitan viabilizar el proceso de vinculación universidad-sector productivo. Para ello se realizó una investigación de campo de carácter descriptivo-exploratorio, aplicada y no experimental. La población estuvo constituida por 22 dependencias de LUZ, que desarrollan actividades de vinculación con el sector productivo. El instrumento utilizado para recabar información, estuvo conformado por un cuestionario. Los resultados, fueron analizados estadísticamente. Se concluye que la vinculación entre la universidad y el sector productivo está condicionada por la falta de política para ello y la desconfianza del sector productivo, así como por el entrabamiento de la normativa universitaria. Se elaboran propuestas estratégicas de gestión tecnológica para aplicarlas a las dependencias universitarias de LUZ y viabilizar el proceso de vinculación universidad-sector productivo.

Palabras clave: Universidad del Zulia, sector productivo, gestión tecnológica, lineamientos estratégicos.

Recibido: 02-09-03 . Aceptado: 03-05-26

* Profesora Titular de la Universidad del Zulia. Facultad de Ciencias Jurídicas y Políticas. Escuela de Trabajo Social. Directora del Centro de Investigaciones en Trabajo Social. Acreditada en el PPI. Magíster en Gerencia de Empresas. Doctora en Ciencias Gerenciales.

E-mail: cymart@hotmail.com

** Profesora Titular de la Universidad del Zulia. Facultad de Ciencias Jurídicas y Políticas. Escuela de Trabajo Social. Secretaria Docente de la Escuela de Trabajo Social. Acreditada en el PPI. Especialista en Andragogía. Doctora en Ciencias de la Educación.

E-mail: tsmaril@hotmail.com

*** Becaria Docente adscrita al Centro de Investigaciones en Trabajo Social. Facultad de Ciencias Jurídicas y Políticas. Escuela de Trabajo Social. La Universidad del Zulia. Licenciada en Trabajo Social.E-mail: rligibther@hotmail.com 


\section{Technological Management in the University of Zulia-Productive Sector Relationship}

\section{Abstract}

The purpose of this study is to provide proposals for technological management, which applied to the different University of Zulia dependencies, will allow for the viability of a university-productive private sector relationship. In order to do this descriptive-exploratory applied and non-experimental field research was carried out. The research population was limited to the 22 University of Zulia dependencies which develop activities that create relationships with the productive sector. The instrument used to collect data was a questionnaire. The results were analyzed statistically. The conclusion is that the University and the private sector are subject to lack of policy in this area, as well as a lack of confidence on the part of the private sector and the need to un-encumber university norms in this area. Strategic proposals were formulated for technological management to be applied in the university dependencies at LUZ, and to facilitate the process of university-productive sector bonding.

Key words: University of Zulia, productive sector, technological management, strategic guidelines.

\section{Introducción}

Actualmente, es imposible desconocer la enorme importancia del avance de la Ciencia y Tecnología para la explicación de procesos vitales del quehacer económico y del desarrollo de las naciones, razón por la cual, se han convertido en herramientas estratégicas. La Ciencia y Tecnología (C y $T$ ), son un cuerpo de conocimientos científicos que han dado lugar; a un fenómeno mundial que se conoce como la Sociedad del Conocimiento la cual requiere para su construcción el desarrollo tanto de la Ciencia como de la Tecnología. No obstante la producción y el uso que se le ha dado al conocimiento científico y tecnológico ha sido diferente en las naciones, y por esta razón, su grado de desarrollo es desigual. En los países desarrollados, el conocimiento se sustenta en una estrategia de desarrollo de recursos humanos y en el fortalecimiento de las capacidades para crear, generar, difundir, asimilar y usar el conocimiento científico y tecnológico.

Así pues, la educación del recurso humano y el desarrollo de sus capacidades se constituyen en la base fundamental de cualquier estrategia de desarrollo y competitividad. De allí, la necesidad de repensar a las universidades ante los cambios que depara el nuevo contexto social del siglo XXI, caracterizados por la globalización, competitividad, y acelerados cambios tecnológicos.

Dentro de esa realidad, se deriva que el desarrollo de las naciones está centrado en dos aspectos fundamentales que son: la calidad de la educación y la investigación como eje fundamental en la producción de conocimientos. En ese sentido, la educación y la investigación son consideradas dos fuerzas dinámicas que interactúan y se complementan en un proceso significativo de aprendizaje participativo entre docentes y estudiantes, orientado a interpretar la realidad dentro 
de un contexto social. Por lo cual se hace necesario promover la investigación entre la universidad y el sector productivo (U-SP) en torno a objetivos y metas colectivas claras, donde se involucre la superación de las percepciones mutuas, priorización de la educación superior como instrumento de desarrollo, el establecimiento de una dirección bidireccional entre la U-SP y el incremento de la calidad del producto universitario.

Actualmente las universidades venezolanas tradicionalmente se han vinculado al sector productivo sólo mediante la provisión de los recursos humanos calificados y debido a la competencia en mercados internacionales menos protegidos y sujetos a la ley del más fuerte, el sector productivo se ha visto en la necesidad de requerir de las universidades conocimientos y técnicas que puedan incorporarse a la producción de bienes y servicios. Esta demanda del sector productivo a las universidades del país, ha provocado la necesidad de establecer un vínculo creciente entre estos dos sectores de la sociedad, para colaborar en la ejecución de programas y proyectos de investigación y desarrollo (I+D) (Solleiro, 1993:27).

Arocena (1997:36), señala que las fronteras entre la ciencia y la tecnología cada día son menos visibles y que es imposible pensar en los problemas de la ciencia y de la tecnología de manera separada. A raíz de esa nueva visión de la ciencia y la tecnología, el conocimiento adquiere un nuevo papel estratégico como factor principal de producción y riqueza, de manera que solamente los grupos sociales que los posean tendrán en sus manos el control de las sociedades. Tal revalorización del conocimiento ha generado un reto relacionado con la pertinencia de la ciencia y tecnología en el acontecer social, económico y cultural que demanda una racionalidad distinta para manejar la inventiva y la creatividad en el presente.

Ante el nuevo papel del conocimiento, las universidades adquieren un papel protagónico, al ser el centro de mayor concentración de potencial de investigación, y por lo tanto se convierten en instrumentos fundamentales en las que se apoya la sociedad e impulsan un crecimiento económico sostenido.

En referencia a lo anterior, Arocena (1997:42), establece que una Universidad es una institución en la cual la enseñanza y la investigación están íntimamente ligados, y son cultivadas con igual ahínco; por lo que la universidad que no realiza investigación no es universidad. Es por eso, que en la medida en que se realice mayor investigación, ello redundará positivamente tanto en el avance de los conocimientos y de sus aplicaciones como en la calidad de la enseñanza.

Al mismo tiempo, el citado autor subraya que el nuevo papel del conocimiento ha generado un "continuo Ciencia-Tecnología-Educación" que desempeña una función medular en el mundo contemporáneo, cuyos componentes no pueden ser consideradas de manera separadas ya que existen múltiples relaciones reciprocas entre ellas.

Dentro de este "continuo CienciaTecnología-Educación". la Ciencia le suministra a la Tecnología los esquemas de comprensión del mundo real reflejados en publicaciones, y la Tecnología aplica los avances de la Ciencia al servicio de la humanidad reflejados en bienes y servi- 
cios. Desde esta perspectiva, se asume que "el avance de la ciencia tiene lugar en el contexto de aplicación más bien que en el contexto de descubrimiento" (Arocena, 1997).

De allí pues, en el mundo de hoy, las universidades como medio educativo deben impulsar sus tareas irrenunciables no sólo en el aspecto de formación, sino investigación y extensión que haga realidad el "continuo Ciencia-TecnologíaEducación".

Al respecto, Piñero (1998:15), señala que debe darse atención a la gestión integral de la educación en y para la Ciencia y Tecnología ( $C$ y $T$ ) en los centros educativos con la finalidad de generar una cultura científica y tecnológica alimentados de la investigación "como principio educativo"; por ello los esfuerzos en la educación (con especial énfasis a nivel superior) deben orientarse en la adquisición de nuevos conocimientos y en el desarrollo de habilidades actualizadas a partir del acercamiento deliberado y explícito del mundo educativo, tecnológico y productivo.

Para ello, es necesario replantear el papel de las Universidades a fin de cumplir con la misión de formar recursos humanos altamente capacitados responsables entre sí y críticamente consciente, cuyo propósito fundamental sea la producción de conocimientos que satisfagan las necesidades socioeconómicas del entorno inmediato y del país.

En tal sentido, es fundamental el papel que juega la gestión tecnológica, la cual ha sido considerada como la gerencia sistemática del proceso de generación de nuevos conocimientos, y como la gerencia del desarrollo del conjunto de destrezas e instrumentos necesarios para sostener un programa pertinente y sistemático de mejoras del proceso productivo capaz de conducir a la generación y captación de nuevo conocimiento, la cual considera un sistema constituido por cuatro dimensiones: El mercado tecnológico, la transferencia tecnológica, la protección legal del conocimiento y la negociación tecnológica.

La cooperación entre la universidad y el Sector Productivo en muchos países se ha visto como poco importante para el éxito económico y comercial. Los vínculos más relevantes entre la academia y las empresas, como se sabe, son el entrenamiento y la provisión de capacidad científica y tecnológica y la comercialización de los resultados de la investigación. Sin embargo, está claro, como resultado de muchos ejemplos de cooperación efectiva entre la academia y el mundo de los negocios, en temas de importancia estratégica, que los beneficios pueden ser muy importantes.

\section{La Gestión Tecnológica}

La Gestión Tecnológica, tiene diversos sentidos según diferentes autores; varios de ellos los conciben como un proceso, aunque difieren en su enfoque. Para unos la Gestión Tecnológica es un proceso que permite el manejo de la tecnología desde su desarrollo, otros la ven como un proceso integrador de funciones y otros como un proceso de desarrollo de implantación del enfoque estratégico (Medellín, 1994:96).

Paredes (1991:58), conceptualiza la Gestión Tecnológica como el conjunto de actividades estratégicas de carácter 
técnico-gerencial que se implementa en una organización productiva para orientar el manejo y control de la variable tecnológica, a través de procesos y metodologías que sirven para mejorar la productividad de la organización a los fines de maximizar sus resultados.

De igual forma Paredes (1996:29), plantea que en cuanto a la gestión empresarial el desarrollo tecnológico exige concentrar esfuerzo en el área técnico productiva, principalmente buscando como objetivo una mayor estandarización de las normas técnicas, mejorar la calidad productiva y fortalecer estrategias de reducción de costos. En el área de mercado y negociación es requisito indispensable la investigación de los factores que ofrezcan una mejor información acerca de las necesidades de los clientes así como también inducir la participación de las empresas en ferias y exposiciones de productos en el plano nacional e internacional.

Paredes (1996:32), acota que esta gestión involucra tanto la búsqueda, el desarrollo y la introducción de un nuevo producto o proceso de producción en una empresa manufacturera privada, como la racionalización de la organización, la dirección y el control de un servicio público tal como el de salud o de una unidad gubernamental, tal como el municipio. En otras palabras, la gestión tecnológica se preocupa tanto de la innovación en tecnologías duras, como las relativas a la creación y el desarrollo de nuevos productos (bienes y servicios) y procesos físicos o químicos; como de la innovación en tecnologías blandas como las relativas a nuevas maneras de organizar, dirigir y controlar las actividades de la organiza- ción, sea ésta privada, pública o de economía mixta. La gestión tecnológica es una de las actividades claves de la empresa ya que le permite servir en forma eficiente y eficaz a sus clientes, enfrentar en forma dinámica a sus competidores tanto internos como externos, mantener un ambiente creativo y participativo de trabajo y garantizar una rentabilidad económica atractiva en el largo plazo.

Por su parte, Camacho (1998:112), señala que para que una nación pueda ser capaz de implantar una gestión tecnológica que materialice la innovación debe procurarse la existencia de los siguientes elementos: existencia de una demanda para la innovación nacional, existencia de una cultura innovadora individual e institucional que desarrolle capacidades tecnológicas y capacidad para atenuar los riesgos inherentes a dicha innovación.

Según este mismo autor, la innovación tecnológica adquiere importancia dentro del manejo tecnológico fundamentalmente en razón de:

- La globalización de las economías que configura una nueva situación socioeconómica en el mundo.

- La capacidad innovadora es el requisito básico del liderazgo económico.

- El núcleo del proceso innovador es la empresa.

La gestión tecnológica utiliza procesos tales como el mercadeo tecnológico, transferencia tecnológica, protección legal del conocimiento y negociación tecnológica, los cuales desglosaremos a continuación, y que son los que van a permitir la vinculación entre la universidad con el sector productivo. 


\subsection{Mercadeo Tecnológico}

El mercadeo de un negocio es un factor esencial en su supervivencia. Para llevar a cabo una dirección exitosa, necesita planear con cuidado, identificar los resultados requeridos y pensar en organizar y controlar las actividades que harán realidad estos resultados. Al final de cada proceso de mercadeo, se impone una revisión del éxito logrado y cómo se pueden obtener mejores resultados en el siguiente período. De esta manera, se entiende mejor el potencial del negocio, se adquiere más confianza y se aumenta el poder para competir y sobrevivir.

Dentro de lo que es el proceso de planeación de mercadeo, se busca responder cuatro preguntas importantes: ¿Qué venderá?, ¿A quién se lo venderá?, ¿Cuánto venderá?, ¿A qué precio venderá?

El plan de mercadeo obtiene las respuestas siguiendo una secuencia lógica: misión de la empresa, ¿Hacia dónde quiere que vaya el negocio?; análisis externo: ¿Qué oportunidades y dificultades hay en su camino?; análisis interno: ¿Cuáles de sus puntos fuertes y débiles influyen en la consecución de este objetivo?; objetivos de estrategia: ¿En qué mercados competirá y con qué base?; operaciones de mercadeo: ¿Cuál será el papel que desempeñará cada elemento de la mezcla de mercadeo?; programación: ¿Cómo se asegurará que ocurran las actividades necesarias en la secuencia correcta y en el momento oportuno?; presupuesto: ¿Cuánto costarán las operaciones de mercadeo?

Por lo tanto, el plan de mercadeo consiste en usar información del presente para darle forma al futuro.

\subsection{Transferencia Tecnológica}

La transferencia de tecnología puede ser definida según ALTEC (1992), como el traspaso de un paquete tecnológico o parte de él, desde una unidad u organización hacia otra, con el objeto de que esta última produzca y distribuya bienes y servicios.

La transferencia de tecnología en forma material y, progresivamente, en forma no material, desempeñará un papel cada día más determinante, según las tendencias, en las relaciones de comercio exterior de los países. Abarca la transferencia de tecnologías nuevas y conocidas, de tecnologías altamente desarrolladas y de tecnologías estándar.

Para determinar el nivel de transferencia de tecnologías debe considerarse según Pride y Ferrell (1993:129), sobre todo, su carácter complejo. No es justo considerar la transferencia de tecnologías sólo desde el ángulo de las tecnologías nuevas y sofisticadas, y pasar por alto el amplio campo de transferencia de tecnologías conocidas y probadas.

De acuerdo a los planteamientos de la CEPAL-ILPES y Otros (1993), simultáneamente surgen, hoy como antes, nuevos campos de aplicación para tecnologías conocidas que también puede clasificarse como tecnologías estándar. Eso no sólo se refiere al sector de los países en vías de desarrollo sino que es un problema de trascendencia internacional. Por eso, limitar la discusión a un nivel "elitista" de las tecnologías nuevas y sofisticadas no responde a las realidades de la economía internacional. 


\subsection{Protección Legal del Conocimiento}

A escala superior, la educación presenta como premisa, servir de órgano formador, creador y transformador de conocimientos y pensamientos que permitan a la sociedad el enriquecimiento y desarrollo de sus dimensiones, y promover los cambios que ella requiera para su propio beneficio y crecimiento. Cumpliendo las Universidades Nacionales dentro de su proceso de desarrollo un rol determinante, de profesionales críticos, promotores $\mathrm{y} / \mathrm{o}$ lideres de cambios estructurales.

La importancia del conocimiento que se genera en las universidades por las actividades de investigación que promueven aportes intelectuales, permite que la propiedad intelectual se presente como un instrumento para la efectiva transferencia de estos hacia el aparato productivo.

La propiedad intelectual, pues representa la herramienta que permite promover un efectivo sistema de protección, comercialización y transferencia de tecnologías.

La comercialización y transferencia de las tecnologías generadas por la investigación universitaria al sector industrial, además de convertirse en un objetivo importante, es fundamental que la transferencia de estos resultados cuenten con los mecanismos legales que permita una efectiva negociación y explotación.

El uso del sistema de protección hacia la propiedad intelectual, debe difundirse para pasar a ser parte de todo el colectivo, de forma ilimitada y para el beneficio de la humanidad todos sus conocimientos y desarrollos tecnológicos. Pero esta premisa, pierde su sustento cuando se evidencia que los empresarios para explotar o comercializar un producto desean contar con los derechos exclusivos sobre la misma, esta actitud responde a la necesidad de contar con una ventaja competitiva en el mercado frente a sus competidores.

Otro aspecto a considerar sobre la necesaria protección del nuevo conocimiento o tecnología producida por los universitarios, es que el sistema de la propiedad intelectual, protege igualmente a sus creadores; reivindicándoles la paternidad como autores e inventores; y más aún, la regulación prevista por algunas universidades y organismos del Estado, les recompensa con reconocimientos académicos, públicos y económicos.

La actividad de proteger y transferir conocimientos a través de la propiedad intelectual, aumenta la manera y probabilidad de que la sociedad tenga acceso a nuevos productos y servicios inventados o descubiertos por investigadores universitarios.

Hoy en día, la mayoría de las universidades de los países desarrollados cuentan con políticas en materia de propiedad industrial, al igual que con definidas estrategias de cómo proteger sus desarrollos, comercializarlos y transferirlos; igualmente, cuentan con la plataforma organizacional, de procedimientos y de normas, que le permite a su personal conocer y comprender el alcance y pertinencia en su trabajo, como también saber obtener los respectivos beneficios o privilegios que se deriven. Por último, es oportuno citar las palabras de Lita Nelsen, Directora de la Oficina de Licencias de Tecnologías del Massachusetts Institute of 
Technology (MIT) citado por Sánchez (2000:142): "la nueva importancia de la propiedad intelectual en al ámbito universitario, refleja el cambio hacia una nueva visión y concepción de la vinculación de la investigación universitaria, orientada a estar cerca de la sociedad.

\subsection{Negociación Tecnológica}

Vasconcellos (1992:258), afirma que es indispensable que los centros de Investigación y de Desarrollo de las empresas realicen un esfuerzo por vincularse con estos centros académicos, para contemplar sus capacidades físicas y humanas, al mimo tiempo, superar los inconvenientes de la falta de experiencia en la generación de conocimientos útiles a las organizaciones productivas.

Para Cadenas, citado por Fernández y Fernández (1997:98), señala que la vinculación exige actividades profesionales de negociación, a fin de asegurar dos puntos relevantes: que los intereses de la organización productiva sean compatibles con los objetivos planteados para el trabajo conjunto y; que las organizaciones académicas puedan mantener equilibrio entre su misión de docencia e investigación y la generación compartida de paquetes tecnológicos para la industria.

De acuerdo a lo que plantea Ertel (1996:73) existen diferentes estrategias a aplicar en el proceso de negociación. La estrategia ganar - ganar se centra la atención en aportar la mayor cantidad de alternativas de solución a los problemas de la contraparte. Interesándose en la óptima atención a las necesidades de ésta y con ella coparticipa, como si se tratase del inicio de una sociedad con propósitos comunes. Se proponen contraprestaciones justas, llegando, en ocasiones, a que sólo cubran los gastos derivados del bien o servicio prestado y se obtengan utilidades marginales.

De lograrse lo anterior, tanto las organizaciones participantes como los negociadores resultarán beneficiados. Todos verán satisfechas sus expectativas y el proceso se desarrollará en un ambiente relajado y cordial. Las organizaciones reconocerán a sus miembros como elementos identificados con sus propósitos y valorarán la labor, de acuerdo con los sistemas que para ello tengan establecidos.

El negociador de tecnología debe manejar conocimientos sobre el proceso de innovación tecnológica: conformación y contenido de paquetes tecnológicos; el proceso de negociación, técnicas de creatividad, conducción de reuniones, liderazgo; la planificación de proyectos por etapas y su consecuente industrialización integral; el entorno industrial y económico; patentes y otras figuras de propiedad industrial, integración de documentos, estrategias de patenteamiento, vigencia y otros datos relevantes. Contando con conocimientos sobre técnicas y tácticas de negociación, es posible emplearlos en beneficio de los representados.

Las negociaciones alcanzarán su punto culminante cuando produzcan contratos equilibrados, en los que se prevean todas las posibles razones de conflicto ofrezcan las fórmulas necesarias para ventilar controversias, sin perjuicio de los contratantes. De esta manera garantizarían las relaciones de largo plazo entre las organizaciones miembros de la cadena institucional, favoreciendo la generación de tecnología. 
La negociación, por lo tanto, ofrece al centro cautivo la posibilidad de realizar sus funciones de acuerdo con los planes y programas establecidos, le permite vincularse con otros para completar sus capacidades y le proporciona la forma de articular sus resultados con el quehacer industrial cotidiano.

\section{Vinculación Universidad-Sector Productivo}

La función primaria de la gestión de vinculación es el desarrollo de objetivos comunes, los cuales serán la guía del proceso de vinculación. Normalmente, estos objetivos se refieren al desarrollo de una investigación o a la transferencia de una tecnología.

A continuación se describen algunas de las funciones del Centro para la Innovación Tecnológica (CIT) de la Universidad Nacional Autónoma de México (UNAM), las cuales se apegan a las necesidades de la vinculación en países latinoamericanos (Solleiro, 1993:115).

- Búsqueda de información técnica y económica relacionada con los proyectos.

- Establecimiento de estrategias de protección industrial y redacción de patentes cuando corresponde.

- Colaboración con el investigador en la orientación del proyecto, para enfocarlo adecuadamente a las necesidades de la industria.

- Búsqueda y vinculación con empresas interesadas en la tecnología.

- Redacción y negociación de contratos de transferencia tecnológica.

- Seguimiento de los proyectos una vez contratados, para disminuir las barreras de lenguaje y costumbres entre investigadores y empresarios.

- Gestiones para obtener financiamientos.

- Búsqueda y contratación de consultores especializados.

- Realización de perfiles de mercado y de factibilidad técnico-económico.

- Desarrollo de la función de mercadotecnia de servicios (Solleiro, 1993).

Un factor crucial para el éxito o fracaso de la vinculación lo constituye el desempeño y las actitudes de las personas involucradas en el proceso de vinculación. El líder industrial debe comprometerse en un diálogo constructivo con el líder académico para entender la misión, las actividades y las limitaciones de la universidad. Por su parte, el líder académico necesita prepararse para entender el mundo de los negocios, su misión lucrativa, las presiones para generar proyectos rentables, la inevitable tendencia a enfocar el corto plazo para asegurar la viabilidad empresarial a largo plazo y la necesidad de trabajar en condiciones competitivas (Solleiro, 1993:120).

El éxito dependerá en gran medida de que ambos líderes se entiendan y acuerden realizar algunas cosas conjuntamente. Entonces, ellos deberán tratar de influenciar a sus colegas de sus respectivas organizaciones para lograr que las actividades se realicen. Asimismo, ellos deberán establecer parámetros y criterios de evaluación y un sistema de estímulo y reconocimiento para aquellos que contribuyan al establecimiento de la vinculación (Blais, 1991:69).

La vinculación Universidad-Sector Productivo puede adoptar diversas modalidades; donde el desarrollo y la trans- 
ferencia de tecnología son oportunidades para viabilizar dicha vinculación. Se puede empezar por la prestación de servicios especializados y programas de capacitación, posteriormente, pueden hacerse algunos contratos de consultoría y asistencia técnica, en áreas donde la universidad tenga un alto nivel de competencia. Finalmente, se podrá pasar a las fases superiores relacionadas con la investigación. Tal vez, el área de mayor oportunidad inmediata para muchas universidades latinoamericanas sea la vinculación vía la formación de recursos humanos y la educación continua. El ritmo del cambio tecnológico actual hará que los profesionales queden obsoletos en plazos cada vez más breves. Este hecho impone al sector productivo entrar en la filosofía de aprendizaje continuo, que le permita revitalizar y mantener sus cuadros humanos. En este aspecto las universidades pueden tener una gran incidencia a corto plazo (Macaya, 1992:19).

Una vinculación efectiva entre la universidad y la industria involucra aspectos relativos a patentes y licenciamiento. Para ello, es necesario poseer una capacidad de innovación local (universidades, institutos y empresas) para evitar de este modo la dependencia tecnológica externa. Esta capacidad debería estar asociada a recursos de alta calificación, a una infraestructura apropiada de laboratorios a recursos financieros y a la existencia de un ambiente institucional, de creatividad y de líneas de investigación y desarrollo relevantes para el desarrollo nacional presente y futuro (Paredes, 1996:30).

En este sentido, la Universidad del Zulia, se propone constituirse en genera- dora de respuestas pertinentes con base en resultados de una educación con calidad científica y pertinencia social, diseñando, e instrumentando alternativas viables, que fortalezcan los procesos de cambio del país (Consejo Universitario, 1994).

El requerimiento de LUZ para su vinculación con el sector productivo, propicia el impulso a la generación de mayores ingresos a través de la comercialización de sus bienes y servicios, de negociaciones con el sector productivo en cuanto a la creación y adaptación de conocimiento, de incremento patrimonial mediante el desarrollo inmobiliario de la ciudad universitaria.

Exigencia sentida de la comunidad de que los centros de investigación orienten sus esfuerzos a la generación de conocimientos aplicados al desarrollo de bienes y servicios de calidad, lo cual a su vez, permitiría la generación de ingresos propios para La Universidad del Zulia.

Con base en los aspectos teóricos que sustentan la investigación y en función de los objetivos de la misma, se emiten las siguientes aseveraciones, en cuanto a las dimensiones que se derivan de la Gestión Tecnológica.

\section{a) Derivadas de la Dimensión Mercadeo Tecnológico}

- El objetivo fundamental asociado a la estrategia de mercadeo tecnológico se fundamenta en la proyección de la universidad en el medio productivo y en la búsqueda de generación de ingresos propios.

- Los contactos previos con potenciales clientes, el conocimiento de la cartera de producto $y / 0$ servicios son aplicadas como acciones estratégicas de mercadeo. 
- La ética empresarial, la promoción y la publicidad, la planificación del precio, representan las funciones de mercadeo aplicadas por las dependencias universitarias para vincularse con el sector productivo.

- No existen políticas universitarias establecidas en cuanto a mercadeo tecnológico.

- Existe desconocimiento del potencial de la oferta tecnológica universitaria.

- Se hace presente la falta de integración entre las dependencias universitarias y el sector productivo.

- El procedimiento de mercadotecnia ejecutado por las dependencias universitarias resulta inadecuado.

- Se hace necesario difundir en forma continua y sistemática el trabajo y los logros alcanzados por la universidad para conocimiento de los potenciales usuarios.

\section{b) Derivadas de la Dimensión}

\section{Transferencia Tecnológica}

- Los canales de transferencia tecnológica que han logrado ser transferidos al sector productivo con mayor éxito son la asistencia técnica, los cursos de capacitación, las asesorías, la consultoría y resultados de proyectos de investigación.

- Las alianzas estratégicas con el sector productivo y el estímulo-beneficios económicos para el personal universitario mas la existencia de dependencias universitarias para la transferencia son factores claves que inciden en la transferencia de tecnología hacia el sector productivo.

- Las dependencias universitarias no han desarrollado estructuras internas para la transferencia de tecnología, siendo éstas un factor clave para ello.

- El entrabamiento de la normativa universitaria y la ausencia de políticas de transferencia tecnológica se constituyen en elementos que obstaculizan el proceso de transferencia tecnológica de la universidad hacia el sector productivo.

- La burocracia universitaria y la falta de personal especializado no han permitido una mayor viabilidad del proceso transferencia tecnológica.

- No existen políticas universitarias definidas en cuanto a transferencia tecnológica.

c) Derivadas de la Dimensión Protección Legal del

\section{Conocimiento}

- Las dependencias universitarias efectúan acciones de protección legal del conocimiento científico-técnico fundamentalmente en la protección de la biotecnología así como también los contactos con dependencias universitarias responsables de la materia y con organismos gubernamentales.

- La solicitud de patentes, derecho de autor y de diseños industriales se asocian con la protección legal del conocimiento generada en la universidad.

- La defensa de la autoría intelectual, el estímulo a la innovación tecnológica, el prestigio e imagen de la universidad y los beneficios económicos de inventores se encuentran asociadas con la protección legal.

- Se reconoce el Consejo de Fomento como el organismo responsable de la protección legal del conocimiento generado en la universidad. 
- Se destaca la ausencia de políticas de propiedad industrial y en general de protección legal del conocimiento.

- La normativa universitaria se caracteriza por ser extemporánea.

- Los gestores responsables de ejecutar la vinculación son desconocedores de la materia.

- Se hacen presentes debilidades organizacionales que contribuyen a diluir las relaciones de interdependencia y complementariedad entre la universidad y el sector productivo.

\section{d) Derivadas de la Dimensión Negociación Tecnológica}

- Las acciones gerenciales utilizadas en la negociación tecnológica en el proceso de vinculación son transferencia y difusión de la tecnología universitaria, la comercialización y la formulación de contratos tecnológicos.

- Se encuentran presentes situaciones consideradas como la obtención de beneficios mutuos, las estrategias de mercadeo, la definición de modalidades/canales de transferencia tecnológica y la protección previa del conocimiento científico.

- Existe desconocimiento en la negociación de contratos y en cuanto a la consecución de financiamiento externo.

- Se carece de estructura organizativa para negociar.

Desde un perspectiva general se concluye que las principales modalidades de vinculación de las dependencias universitarias con el sector productivo están representados por los cursos de extensión, los servicios técnicos, la asistencia técnica y las asesorías, de igual manera se detectó que existe desconfianza por parte del sector productivo, falta de políticas para la vinculación y entrabamiento de la normativa universitaria.

En consecuencia, se hace necesario el desarrollo de políticas institucionales de mercadeo, transferencia y negociación tecnológica para fortalecer el proceso de vinculación universidad-sector productivo, ya que el nivel de organicidad interno en las respectivas dependencias universitarias es débil e inadecuado, haciendo especial énfasis en el fortalecimiento de estímulo y reconocimiento académico y económico al personal universitario.

\section{Relación Universidad- Sector Productivo}

Es impostergable delinear una estrategia que implique la concientización de esta vinculación con los involucrados: gobierno-universidad-sector productivo en torno a objetivos y metas colectivas claras en primer lugar y que contemplen en segundo lugar, "la integración conceptual, financiera y operacional de los sectores involucrados.

En este sentido Rojas y Bermúdez (1997) menciona que "esta integración involucra la superación de las percepciones mutuas de suspicacia entre los tres componentes, la priorización de la educación superior como instrumento del desarrollo, el establecimiento de una dirección bidireccional entre la universidad y el sector productivo y el incremento de la calidad del producto universitario".

Para enfrentar el reto y hacia el logro de la vinculación universidad-sector productivo la primera debe convertirse en una institución más dinámica y participativa, con la implementación de mecanis- 
mos que permitan incrementar la productividad en las áreas claves.

La universidad de hoy se puede abocar a estudiar en profundidad las posibilidades de generar recursos a través del trabajo de sus especialistas y del uso de sus múltiples facilidades (investigación, consultoría, educación, licenciamiento, publicaciones, formación de empresas, desarrollo de parques e incubadoras, $u$ otras actividades conexas).

Ello requiere del estudio conjunto con el Sector Productivo para que el trabajo de la universidad responda a las necesidades reales de la empresa.

Se debe aceptar que hoy en día el trabajo de la universidad va más allá de sus funciones tradicionales de docencia, investigación y extensión. Ella puede además llegar a influir poderosamente en los modelos y en las orientaciones del desarrollo económico e industrial, social y cuttural de las regiones donde está inserta.

El Centro Interuniversitario de Desarrollo (1994:23), plantea que se observan cambios profundos en las universidades en todo el mundo en su actitud hacia su vinculación con la industria. Ello es particularmente cierto en Europa y EEUU donde el tema ha alcanzado una relevancia tal que prácticamente no existe universidad que no haya establecido mecanismos para promover la vinculación. Cabe preguntarse qué puede haber ocurrido para que los académicos salgan de sus laboratorios para observar el mundo exterior y visualizar sus eventuales contribuciones y, al mismo tiempo, el Sector Productivo ya no sólo está interesado en los profesionales que ella le provee, sino también en el trabajo académico.

Paredes (1996:57), afirma que el principio dialéctico de la vinculación Universidad-empresa está referido a las contradicciones que se establecen entre las motivaciones que tienden a favorecer la cooperación efectiva entre las partes, con las tradicionales barreras que inciden de diferentes manera en el efecto vinculante. En el marco de estos señalamientos, López (1991:45), identifica las siguientes motivaciones para la vinculación para ambos sistemas:

\section{Universidad}

- Industria como fuente de recursos.

- Exponer los estudiantes al mundo real.

- Mejor formación a los postgrados.

- Autofinanciamiento de ciertas actividades.

- Contribuciones a la sociedad.

- Acceso a las instalaciones industriales.

\section{Industria}

- Tener acceso a recursos humanos.

- Tener acceso a la Ciencia y Tecnología.

- Tener soporte técnico de excelencia.

- Acceso a las instalaciones universitarias.

- Obtener prestigio e imagen.

- Uso de recursos económicos disponibles.

En el marco de lo expuesto se presenta la propuesta de vinculación Universidad-Sector Productivo y la propuesta de vinculación de Gestión Tecnológica Universidad-Sector Productivo. 


\subsection{Plan estratégico de relación universidad sector-productivo}

Se plantea su ejecución a través de la Universidad del Zulia, por atribuciones que se confieren a las universidades venezolanas a través de la Ley de Universidades con la finalidad de desarrollar tres funciones básicas: Docencia-Investigación-Extensión. En el marco de éstas funciones, la universidad puede realizar actividades de vinculación por medio de un conjunto de dependencias entre las cuales destacan: Consejo de Fomento; Consejo de Desarrollo Científico y Humanístico (CONDES); Consejo Central de Extensión; Parque Tecnológico Universitario; Empresas Rentales y Fundaciones de LUZ; todos ellos bajo la coordinación del Vice-Rectorado Académico de LUZ.

La indefinición de un plan institucional para la vinculación es causa y efecto para que las actividades inherentes a éste proceso se realicen de una manera asistemática y ocasional; lo cual se traduce en duplicidad de funciones entre las dependencias que se interesan en las actividades de vinculación con el sector productivo; sin embargo se hace necesario reconocer el interés y el esfuerzo realizado a nivel de ciertas dependencias universitarias por dar repuestas a los potenciales usuarios del sector productivo.

Se requiere por parte de la institución la definición de un enfoque estratégico que facilite la convergencia de los intereses interinstitucionales de las partes, con las acciones operativas de sus mecanismos de vinculación; donde las dependencias involucradas tengan la capacidad de generar la sinergia necesaria para la acción.
Lo expuesto, conlleva a una visión prospectiva que integre los elementos proactivos para la institucionalización de la vinculación, fundamentado en relaciones de interdependencia (necesidades mutuas de los socios) y de complementariedad (desarrollo de actividades mutuas); todo ello dentro de un proceso de cooperación.

El Plan Estratégico de Vinculación Universidad-Sector Productivo se aplicará tomando como referencia las funciones de gestión tecnológica: mercadeo tecnológico, transferencia tecnológica, negociación tecnológica y protección legal del conocimiento, los cuales actuarán como escenarios para la acción en el proceso de vinculación universidad-sector productivo bajo la coordinación del ViceRectorado Académico y con la participación activa e interdependiente de las dependencias universitarias antes descritas, las cuales deben designar un representante en cada una de las dependencias referidas, quienes serán los responsables de impulsar el proceso de institucionalización de la vinculación, teniendo como punto de partida el establecimiento de agendas para iniciar, mantener, consolidar y evaluar modalidades de vinculación entre la universidad y el sector productivo; así como de promover la realización de diagnósticos que permitan detectar los elementos adecuados para fortalecer el efecto vinculante. En este sentido en el Diagrama 1 se muestra gráficamente, el Plan Estratégico de relación entre estos dos sectores, donde se concibe a todas las autoridades rectorales como el núcleo o eje vinculante con ciertas dependencias universitarias, todo ello concatenado con las cuatro dimensiones establecidas por la gestión tecnológica. 


\section{Diagrama 1 \\ Plan estratégico de relación Universidad-Sector Productivo}

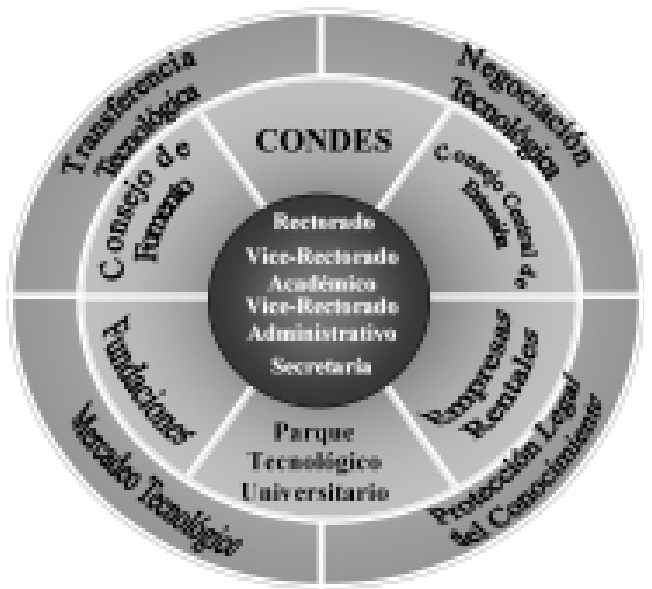

Fuente: Elaboración propia.

\subsection{Plan estratégico de relación Gestión Tecnológica- Universidad- Sector Productivo}

A la luz de los planteamientos expuestos, se procede a presentar el Plan Estratégico de Vinculación de Gestión Tecnológica Universidad-Sector Productivo, del cual se mencionan a continuación las propuestas a seguir por el ViceRectorado Académico y las Dependencias Universitarias (Consejo de Fomento; Consejo de Desarrollo Científico y Humanístico (CONDES); Consejo Central de Extensión; Parque Tecnológico Universitario; Empresas Rentales y Fundaciones de LUZ):

- Formulación de políticas institucionales para el desarrollo de los procesos de gestión tecnológica a saber: mercadeo tecnológico, transferencia tecnológica, negociación tecnológica y protección legal del conocimiento científico-técnico; que permitan viabilizar el proceso de vinculación universidad-sector productivo.

- Fortalecer los aspectos intrainstitucionales estableciendo escenarios de vinculación con base a capacidades internas de las dependencias universitarias.

- Revisión exhaustiva de la normativa universitaria para lograr el desentrabamiento de la misma.

- Creación de la estructura organizativa adecuada.

- Preparación a nivel gerencial de personal especializado y constitución de un equipo promotor y asesor de vinculación de las dependencias universitarias con el sector productivo.

- Establecer y practicar contactos exploratorios con el sector externo, identificando aliados y competidores; realizando revisiones técnicas e interinstitucionales. 
- Analizar las políticas gubernamentales y las políticas del sector privado relacionadas con el proceso de vinculación.

- Propagar y difundir en forma sistemática el trabajo y los logros alcanzados por la universidad para conocimiento de los potenciales usuarios.

- Establecer un sistema de monitoreo ex-ante; en; ex-post; que permita detectar los avances, logros, obstáculos, impacto, con la finalidad de reforzar los aciertos y de aplicar correctivos para lograr la retroalimentación requerida en el proceso de vinculación universidad-sector productivo para que lleve a cabo la evaluación necesaria.

- Establecer indicadores de análisis de impacto en los diferentes escenarios de vinculación, para desarrollar un análisis comparativo.

- Registrar y crear la memoria corporativa y desarrollar los sistemas de información gerencial con la finalidad de detectar las situaciones pasadas y actuales, con miras a desarrollar una visión prospectiva en el proceso de vinculación universidad-sector productivo. De igual manera se ilustra en el Diagrama 2 este plan, el cual se concibe del mismo modo que en el anterior, las cuatro autoridades rectorales como eje vinculante con las dimensiones de la Gestión Tecnológica tales como: Transferencia Tecnológica, Negociación Tecnológica, Mercadeo Tecnológico y Protección Legal del Conocimiento.

\section{Diagrama 2 \\ Plan estratégico de relación Gestión Tecnológica-Universidad- Sector Productivo}

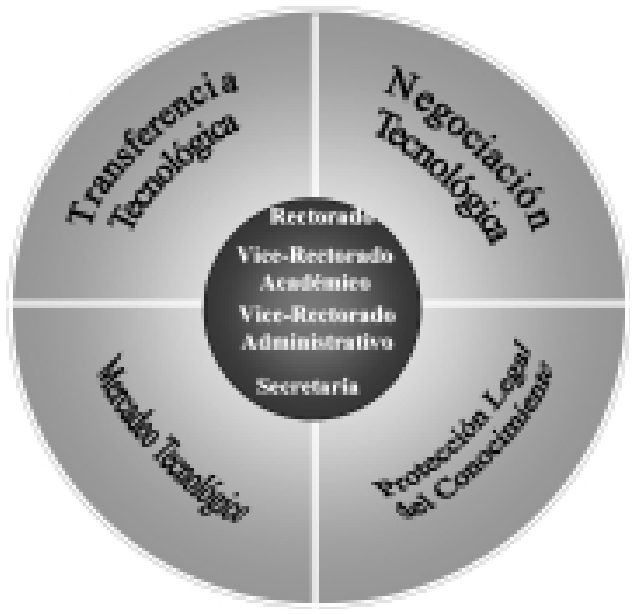

Fuente: Elaboración propia. 


\section{Referencias Bibliográficas}

Arocena, R. (1997), Alternativas de la Educación Superior ante los Escenarios Posibles de la Región: Algunas Ob. servaciones para la Decisión En: La Educación Superior en el Siglo XXI. Visión de América Latina y el Caribe. Cresal/UNESCO. Caracas. Venezuela.

Asociación Latinoamericana de Gestión Tecnológica, ALTEC (1992), Aspectos Conceptuales y Metodológicos de la Gestión Tecnológica. Edic. DOLVIA. Caracas, Venezuela.

Blais, R. (1991), Condiciones para una Cooperación Eficaz Universidad-Industria: un Curso sobre Vinculación Universidad-Sector Productivo. Protec-CIT-UNAM. México

Camacho, Jaime (1998), Incubadoras o Viveros de Empresas de Base Tecnológica. La Reciente Experiencia Europea como Referencia para las Actuales y Futuras Iniciativas Latinoamericanas. Documento presentado en el XII Congreso Latinoamericano sobre espíritu empresarial. Costa Rica.

Centro Interuniversitario de Desarrollo, Programa de las Naciones Unidas para el Desarrollo (1994), Universidad-Sector Productivo. Nuevas formas de Vinculación (Parques Tecnológicos e incubadoras). CINDA, ONU. Mimeografiado.

CEPAL-ILPES/UNESCO/UNU/CYTED-D. (1993), Estrategias, Planificación y Gestión de Ciencia y Tecnología. Venezuela.

Congreso de la República de Venezuela (1970), Ley de Universidades. Gaceta Oficial No. 1.429. Extraordinario. Caracas, Venezuela.

Consejo Universitario (1994), Filosofía de Gestión de la Universidad del Zulia.
Universidad del Zulia. Maracaibo-venezuela.

Ertel, Danny (1996), Negociación 2000. Edit. Mc. Graw Hill. Colombia.

Fernández, Omar y Fernández, Marina (1997), Hablemos de Comercialización de Tecnología. FUNDACITE. Maracaibo, Venezuela.

López Medellín, Solleiro (1991), La Vinculación Universidad-Sector Productivo: Motivaciones y Barreras. UNAM. México.

Macaya, Gabriel (1992), Vinculación de la Investigación Universitaria con el Sector Productivo. Protec-CITUNAM. México.

Medellín, Enrique (1994), Evaluación de la Gestión Tecnológica desde la Perspectiva Universitaria. Memorias del Seminario Internacional de Postgrados en la Planificación, Gestión y Estudios de la Ciencia y Tecnología. UNAM, México.

Paredes, Leopoldo (1996), Una contribución Teórico-Metodológica al estudio de la Gestión Tecnológica. FUNDACITE. Maracaibo, Venezuela.

Paredes, Leopoldo y Bermúdez, Oladys (1996), Aplicación de la Técnica de Escenarios al Estudio del Proceso de Vinculación Universidad-Industria. FUNDACITE. Maracaibo, Venezuela.

Pride W. y Ferrell O. (1993), Marketing, Decisiones y Conceptos Básicos. Edit. Mc. Grae Hill/Interamericana. México D.F.

Rojas, Luis, Bermúdez Lily (1997), Aproximación teórica para el estudio de la comunicación como factor estratégico en la relación LUZ-Sector Productivo. Revista Venezolana de Gerencia. La Universidad del Zulia. Año 2 №3. P. 93-103. Maracaibo-Venezuela. 
Piñero, M. (1998), Educación, Ciencia, Tecnología y Esfuerzo Productivo. Una Nueva Dimensión para el Desarrollo de Venezuela. Tesis Doctoral. Doctorado en Ciencias Mención Investigación. Universidad Dr. Rafael Belloso Chacín. Maracaibo. Venezuela.

Sánchez, Antonio (2000), La Propiedad Industrial en Venezuela: el Caso de La Universidad del Zulia. La Universidad del Zulia. Tesis para optar al título de Doctor en Derecho. Maracaibo. Venezuela.
Solleiro, J.L. (1993), Gestión de la Vinculación Universitaria-Sector Productivo un Curso sobre Vinculación Universidad-Sector Productivo. Protec-CIT-UNAM. México.

Vasconcellos, Eduardo (1992), Gerencia miento de Tecnología: un Instrumento para la Competitividad Empresarial. Edit. Edgard Blücher LTDA. Brasil. 